\title{
Bayesian Network Analysis: A New Approach to Diagnosis and Prognosis
}

\author{
Alok Kumar \\ Assistant Professor, Department of Orthodontics \& Dentofacial Orthopaedics, Awadh Dental College \& \\ Hospital, Jamshedpur, India
}

\begin{abstract}
Malocclusion and dentofacial deformities are diseases with multifactorial etiology, where there is a complex interplay of the various etiologic factors resulting in the deformity or malocclusion. Bayesian network, an innovative statistical tool, provides us with a new approach to understand this complex problem. It is a graphical model that encodes probabilistic relationship among variables of interest. When used in combination with statistical technique this graphic model illustrates the causal relationship among different variables and hence can be used to gain understanding about a problem and predict the consequences of intervention. It has been used successfully in various fields like machine learning, bioinformatics, gene expression analysis as well as medical diagnosis. This network provides support for health professionals in analysis of patient data and making decisions regarding diagnosis and prognosis of a disease condition. This article discusses the characteristics of Bayesian network system, there use in clinical practice and the scope of its use in dentistry in general and orthodontics in particular.
\end{abstract}

Keywords:Bayesian Network, diagnosis, dentistry, orthodontics

\section{Introduction}

Malocclusion and dentofacial deformities are multifactorial diseases believed to be due to complex interaction between genetic factors and environmental factors. However it has not been clearly understood how these factors co-relate with each other to produce malocclusion. This is primarily because of two reasons. First is our limited understanding of genetics specially the factors influencing gene expression. Second is the lack of an appropriate "tool" which could co-relate the various etiological factors enhancing our understanding of the disease process thus facilitating diagnosis and prognosis.

The first limitation has been addressed to some extent by completion of the Human Genome Project in 2003 - an international effort to characterize the human genome through a complete mapping and sequencing of its DNA. ${ }^{1}$ However the process of deciphering and understanding the genomic data is still underway and it may take a few years before we actually start applying and benefitting from the discoveries in terms of preventing, intercepting and curing diseases of mankind.

However it is the second limitation which the Bayesian networking analysis promises to overcome. As this graphic model allows us to learn the causal relationship among different variables and hence can be used to gain understanding about a problem and predict the consequences of intervention.

The use of computers to analyse statistical data and assist health professionals in their activities has been studied since the1950s. Ledley and Lusted were the first to address this possibility in their initial work which focussed on the development of a diagnostic system. ${ }^{2}$

Since then researchers have applied different methods to provide clinical applications with knowledge. Dombal et al studied the diagnostic process using Bayesian probability theory. Their system, the Leeds abdominal pain system, used sensitivity, specificity, and disease-prevalence data for various signs, symptoms, and test results to calculate the probability of seven reasons for abdominal pain. In a controlled prospective comparison study, the system's overall accuracy (91.8 percent) was significantly higher than that of the most senior member of the clinical team who saw each case $\left(79.6\right.$ percent). ${ }^{3}$ The Pathfinder systemfor diagnosis of lymph nodes pathology, also a Bayesian system, was built on the foundation set by de Dombal and colleagues. ${ }^{4}$

In recent years Bayesian network has also found application in dentistry to identify people at risk of oral cancer and pre-cancer. Sebastiani P et al identified some potential applications ofBayesian machine learning methods to the genomic analysis of squamous cell carcinomas of the head and neck. ${ }^{1}$ Recently, BN have been applied tothe analysis of relevant literature in implantology. ${ }^{5}$

However there were no studies which applied Bayesian network analysis to orthodontics till Niere $\mathrm{M}$ et al for the first time applied $\mathrm{BN}$ to evaluate the relative role and possiblecausal relationships among various factors affecting the diagnosis and final treatment outcome of impacted maxillary canines. ${ }^{6}$ 


\section{Bayesian Methods}

Classic statistics provides methods to analyse data, from simple descriptive measures to complex and sophisticated models. The available data are processed, and then conclusions about a hypothetical populationof which the data available are supposed to be a representative sample-are drawn. However it cannot incorporate or "learn" from information provided from external resources. ${ }^{1}$

For better understanding lets imagine a situation where we have to guess the outcome of an experiment that consists of tossing a coin. Normally we would believe that there is an equal probability of the coin landing heads or tails. However if we come to know that the coin is biased and there are more chances for it landing heads, how can we take this into account in the analysis of our data? Scientific and medical data are both example of such situation.

The Bayesian method allows us to incorporate this external information into the data analysis process. In this approach the data analysis process starts with a given probability distribution called as the prior distribution. The Bayesian data analysis process consists of using the sample data to update this prior distribution into a posterior distribution analysis process. The basic tool for this updating is a theorem, proved by Thomas Bayes, an 18thcentury clergyman. The role of Bayes' theoremin this approach is so critical that the whole approach is named after it.

The ability to integrate data with external information, a trademark of Bayesian analysis, provides a natural framework for integrating various forms of information available. The intuition behind this approach is that the conclusions of a studycan be used as prior probabilities for another study, with a principled and seamless integration of the flow of information. The power of the Bayesian approach is that an otherwise undetectable linkage between two variables may be established. ${ }^{7}$

\section{Understanding Bayesian Network}

It is a network based on directed acyclic graphs which actually originated from the genetic studies by Sewall Wright (1921), who developed a method called Path Analysis, a recognized ancestor of Bayesian networks. ${ }^{8}$ However the term Bayesian network was coined by Jude Pearl in 1985. ${ }^{9}$ It can help us understand the types of control and dependency relationships among the different variables.

A Bayesian network, belief network or directed acyclic graphical model is a probabilistic graphical model that represents a set of random variables and their conditional dependencies via a directed acyclic graph (DAG). For example, a Bayesian network could represent the probabilistic relationships between diseases and symptoms.

Let's take the example of skeletal class III malocclusion. Suppose there are two factors which can give rise to this condition - anterior cross bite and deficient growth of maxilla. Also suppose that anterior cross bite has a direct effect on the growth of maxilla. Then the situation can be modelled with a Bayesian network. Such a model can then be used to answer questions like - what is the probability of a patient having anterior cross bite given that he has a skeletal class III relationship? Or we can get answer to a more interventional question like what is the likelihood of a patient developing a skeletal class III malocclusion given that he has anterior cross bite?

Bayesian networks (BN) works with the goals of generating hypotheses of possiblecausal relationships among variables and promoting further specific studies (i.e., randomized clinical trials).BN adopt an intermediate approach between statistics and artificial intelligence. A "network" is composed of a "directed acyclic graph" in which stochastic variables are represented by vertices or nodes of the graph, and oriented lines (arrows) represent the relationships among the variables. The arrows relate the variables in such a way that cycles are not permitted, so that, following the arrows, it is impossible to return to a vertex or starting point. The variables from which the arrows start influence those to which they arrive, possibly through a causal relationship. Hence, BNs combine principlesfrom graph theory, probability theory, computerscience, and statistics. ${ }^{6}$

\section{Clinical Applications in Dentistry}

Bayesian network has been utilized in various specialities of dentistry through their application in various Clinical Decision Support Systems or CDSS. There has been research and development ofCDSSs in dentistry for over two decades. ${ }^{10,11}$ These systems have utilized different types of knowledge representation and have addressed several major areas of dental practice. Different modalities and requirements for dental decision support systems are described in articles by White, Benn et al., and Brickley et al. ${ }^{12,13}$

In a comprehensive 1996 review of the literature on decision support applications in dentistry, White identified over thirty decision support systems.He grouped these systems into seven subareas of dentistry: dental emergencies and trauma, orofacial pain (differential diagnosis), oral medicine(management of oral diseases in the neck and head), oral radiology (interpretation of radiographic lesions and automated interpretation of dental radiographs),orthodontics (analysis of facial growth, landmark identification of cephalometric radiographs, and 
treatment planning), pulpal diagnosis, and restorative dentistry (removable partial denture design). White also classified the systems according to the knowledge representation used, including algorithmic, statistical, rulebased, and image processing systems. White described the need to integrate the decision support systems into the practice environment, by providing real-time quality assurance, better support in treatment planning, and improvement of data analysis. ${ }^{12}$

Publications since 1996 have described the development of neural networks in oral surgery,caries management, pre-therapy decisions in patients with head and neck cancer, and intelligent agents for treatment planning. ${ }^{14,15}$

\section{Applications in Orthodontics}

Preventing, intercepting and treating malocclusion and dentofacial deformities remain the goal of orthodontics even today. In spite of the giant strides orthodontics seems to have taken from the days of its inception as a speciality, we still find our knowledge inadequate when it comes to diagnosing and intercepting complex malocclusions of multifactorial etiology. One of the important reasons for this is our inability to understand the interplay of various etiological factors with each other.

Let's take the example of Long Face Syndrome which is characterised by - long and narrow face and often associated with lip incompetence, constricted maxillary arch and an anterior open bite. If we search literature for the etiology of this malocclusion we come across factors like - mouth breathing habit, pressure habits like prolonged thumb sucking or tongue thrusting, decreased masticatory muscle forces or biting forces, genetic predisposition etc. Now, although there is a general agreement about the possible role these factors may play in the development of this malocclusion there appears to be a lack of clear understanding about the interplay of these factors. For example is there an inter-relationship between prolonged thumb sucking habit and decreased masticatory muscle forces resulting in a long face pattern. This is where a tool like Bayesian Network analysis can give us a better understanding of the complex disease process.

Nieri et al were the first to apply Bayesian network analysis in orthodontics to evaluate relative role and possible causal relationship among various factors affecting diagnosis and final treatment outcome of impacted maxillary canine. Their findings not only amplified the existing knowledge on the multifactorial nature of the problem but they also came up with some new and interesting co-relations. According to Bayesian analysis, bilateral impaction was associated with palatal impaction and longer duration of treatment, the pretreatment alpha angle was a determinant for the duration of both orthodontic traction and overall treatment and the post treatment periodontal outcome was not related to pre-treatment radiographic variables that described the intra-osseous position of the impacted canine. ${ }^{6}$

In addition to the above, this study also managed to bring to light the fact that Bayesian Networking System can be effectively used in orthodontics to get a better insight into the disease process thus facilitating better diagnosis and treatment planning.

\section{Conclusion}

There has been tremendous increase in the awareness and expectations of the society to dental health in recent times. This has led to evidence based practice, encouraging health care providers to use the latest evidences in clinical practice and health services. Improving the quality of health care and minimizing errors in practice are the two goals guiding health care practice today. And if we are to meet these goals we need a better understanding of the disease process as well as effectiveness of the current treatment practices. Bayesian network analysis is an invaluable tool which allows us to do that. By offering new research opportunities it can introduce us to new dimensions of understanding of the disease process and by doing so increase the effectiveness of the treatment we render.

\section{References}

[1] Sebastiani P, Yu Y and Ramoni MF. Bayesian machine learning and its potential applications to the genomic study of oral oncology. Advances in Dental Research 2003;17:104-108.

[2] Ledley RS, Lusted LB. Reasoning foundation of medical diagnosis. Science 1959;130:9-21

[3] Dombal FT, Leaper DJ and Staniland JR. computer aided diagnosis of acute abdominal pain. Br Med J 1972;2(5804):9-13

[4] Heckerman D, Horwitz E and Nathwani B. Update on the pathfinder project. In Washington, DC: IEEE Computer Society Press, 1989:203-7

[5] Nieri M, Clauser C and Francheschi D. Randomized clinical trial in implant study: relationship among methodological, statistical, clinical, Para textualfeatures and number of citations. Clinical Oral Implants Res 2007;18:419-31

[6] Nieri M, Crescini A, Rotundo R, Baccetti T, Cortellini P and Prato GPP. Factors affecting the clinical approach to impacted maxillary canines: A Bayesian network analysis. American Journal of Orthodontics and Dentofacial Orthopedics 2010;137:755-62

[7] Vieland VJ, Sheffield V, Wassink T et al. A new genome screen for autism based on the posterior probability of linkage (PPL) and incorporating language based phenotypes finds evidence of linkage to several genomic locations (abstract). Am J Hum Genet 2003; 73(Suppl5): 196

[8] Wright S. Correlation and causation. J Agr Res 1921;20:557-85 
[9] Pearl J. Bayesian Networks: A model of self- activated memory for evidential learning. Proceedings of the seventh conference of cognitive science society, University of California, Irvine CA pp 329-334.

[10] Stheeman SE, Stelt PF and Mileman PA. Expert Systems in Dentistry. Past Performances - future prospects. J Dent 1992;20(2)6873.

[11] Siegel MA, Firriolo FJ and Frankelstein MW. Computer application in oral diagnosis. Dent Clin North Am 1993;37(1):113-31.

[12] White SC. Decision support systems in dentistry. J Dent Educ 1996;60(1):47-63

[13] Brickley MR, Shepherd JP and Armstrong RA. Neural network: A new technique for development of decision support system in dentistry. J Dent 1998;26(4):305-9

[14] Brickley MR, Sheperd JP. Performance of a neural network trained to make third molar treatment- planning decisions. Med Decis Making 1996;16(2):153-60

[15] Benn DK. Applying evidence based dentistry to caries management in dental practice: A computerized approach. J Am Dent Assoc 2002;133(11):1543-8 